\title{
Diacronie
}

Studi di Storia Contemporanea

$N^{\circ} 8,4 \mid 2011$

Le sembianze di Clio: approcci alla storia

\section{Carlo Marsili, La Turchia bussa alla porta. Viaggio nel paese sospeso tra Europa ed Asia}

\section{Luca Zuccolo}

\section{(e) OpenEdition \\ 1 Journals}

\section{Edizione digitale}

URL: http://journals.openedition.org/diacronie/3496

DOI: $10.4000 /$ diacronie.3496

ISSN: 2038-0925

Editore

Association culturelle Diacronie

Notizia bibliografica digitale

Luca Zuccolo, «Carlo Marsili, La Turchia bussa alla porta. Viaggio nel paese sospeso tra Europa ed Asia », Diacronie [Online], $N^{\circ} 8,4$ | 2011, documento 12, Messo online il 29 octobre 2011, consultato il 24 septembre 2020. URL : http://journals.openedition.org/diacronie/3496 ; DOI : https://doi.org/10.4000/ diacronie.3496 


\section{RECENSIONE:}

\section{Carlo MARSILI, La Turchia bussa alla porta. Viaggio nel paese sospeso tra Europa ed Asia, Milano, Università Bocconi Editore, 2011, 292} pp.

a cura di Luca ZUCCOLO *

Ne mutlu Türküm diyene, "felice colui che si dice Turco". Queste poche parole sono la migliore descrizione del testo di Marsili, che raccoglie nelle pagine del suo libro la summa della sua esperienza come ambasciatore italiano in Turchia (2004-2010).

L'esperienza in Turchia, per Marsili, si pone all'apice di una lunga carriera diplomatica iniziata nel 1970 e passata attraverso altri incarichi di rilievo in qualità di console generale a Edimburgo (1984-87), vice capo missione in Germania (1993-98) e ambasciatore in Indonesia (1998-99). Tra gli altri ruoli rivestiti da Marsili, una menzione speciale va fatta relativamente al biennio 2000-2002 quando ricoprì, al Ministero degli Affari Esteri, la carica di direttore generale per gli Italiani all'Estero e le Politiche Migratorie.

Quest'ultimo incarico, unito al suo lungo soggiorno in Turchia, si riflette ampiamente nel presente saggio, in cui l'Italia e l'italianità hanno un posto ed un ruolo privilegiato nella descrizione dei rapporti che la Turchia intrattiene con la vicina Europa.

Il tema dell'adesione all'Unione Europea, infatti, è il filo conduttore di tutta l'opera di Marsili e si trovano rimandi ad esso in ogni capitolo. L'insistenza sul tema dei 
negoziati di adesione, ben descritti nel capitolo ad essi dedicato, non è strumentale, ma serve al lettore per meglio comprendere le numerose riforme che lo Stato turco e le sue parti sociali stanno adottando da tempo nel cammino per diventare una grande potenza mondiale.

Il saggio di Marsili, indirizzato ad un pubblico non esperto ma desideroso di conoscere la complicata realtà turca, non è semplicemente un racconto sulla Turchia, ma è una descrizione della Turchia fatta dall'interno del Paese attraverso l'analisi degli aspetti che la caratterizzano e dei nodi che la relegano ancora, a torto o a ragione, ai margini dell'Europa.

Senza pretendere di fornire soluzioni definitive ma semplicemente esponendo i fatti, l'ambasciatore italiano mette in luce con una scrittura agile e chiara come la Turchia e i Turchi continuino a guardare con grande interesse all'Europa, ma come il "doppiopesismo" europeo indebolisca e raffreddi questo sentimento, allontanando sempre più la Turchia dall'Unione Europea ${ }^{1}$. Una considerazione che si manifesta chiaramente nella conclusione del capitolo dedicato alle relazioni con Bruxelles, in cui senza mezzi termini Marsili sottolinea che «il rischio di perdere la Turchia si è fatto ormai drammaticamente concreto». Secondo l'autore, infatti, «senza un colpo d'ala della politica, di quella vera, dotata di visione e lungimiranza, la più grande occasione di allargamento per l'Unione Europea sarà andata perduta» ${ }^{2}$.

La lungimiranza è proprio il carattere chiave di questo saggio. Marsili, unendo la sua esperienza all'analisi del quadro socio-politico ed economico del grande Paese musulmano propone una descrizione nuova e inedita della Turchia, la quale ci viene descritta in modo diretto attraverso le osservazioni di una persona costantemente in contatto e in relazione con i principali attori socio-politici turchi.

Il saggio si compone di 15 capitoli e ripercorre tutti gli aspetti della società e della politica turca. Dopo un primo capitolo introduttivo, in cui si propone uno "sguardo d'insieme" sul Paese e la sua storia, l'autore propone un'analisi più dettagliata delle questioni centrali della situazione turca. Questa parte si apre con il capitolo II, in cui Marsili descrive la struttura sociale del Paese per poi passare in rassegna, nei capitoli successivi, i temi della condizione femminile, del rapporto con la religione islamica e

\footnotetext{
${ }^{1}$ MARSILI, Carlo: La Turchia bussa alla porta, viaggio nel paese sospeso tra Europa ed Asia, Milano, Università Bocconi Editore, 2011, p. 32.

${ }^{2}$ Ibidem, p. 245.
} 
con le forze armate, della nazionalità, dell'identità e delle minoranze. L'attenzione nei confronti di questi materie si inserisce pienamente nelle nuove correnti storiografiche che propongono un ripensamento della vicenda Ottomano-Turca proprio a partire dai temi e dal vocabolario nazionale-identitario, identificando nelle relazioni sociali e nel loro mutamento un fattore decisivo nella nascita e sviluppo della Turchia contemporanea.

A questi capitoli, che potremmo definire come propedeutici alla comprensione del complesso e poco noto panorama turco, seguono capitoli più specifici, i quali descrivono con chiarezza la situazione di alcuni dei nodi principali che ostacolano la Turchia nella sua corsa verso il futuro.

In una significativa successione Marsili descrive la questione Curda e quella Armena, passando poi ad analizzare la condizione di stampa e mezzi di comunicazione, non lasciandosi sfuggire i temi legati allo sviluppo della società civile turca. L'autore sposta la sua attenzione sui temi politici, descrivendo la classe politica e i partiti turchi, per poi lanciarsi in considerazioni e commenti sulla politica interna ed estera. Uno spazio a parte è dedicato all'analisi della questione cipriota, forse la più spinosa problematica che il governo di Ankara deve affrontare.

Il saggio si conclude con due capitoli significativi. Il capitolo XIV tratta dei rapporti con l'Unione Europea, cercando di proporre un quadro degli stessi equilibrato e scevro da pregiudizi. Un equilibrio di giudizio palesato dai numerosi rimproveri che l'ambasciatore muove ad una politica europea ancora troppo ambigua ed inefficace nei riguardi della Turchia. Infine il capitolo $\mathrm{XV}$, accanto alle tematiche economiche, tratta della presenza italiana e del ruolo che le industrie e le banche italiane stanno svolgendo in Turchia.

Come accennato in precedenza, l'Italia e le relazioni con gli Italiani sono una costante nel testo di Marsili, il quale presenta le relazioni tra il nostro Paese e la Turchia come molto buone, sebbene queste si fermino spesso esclusivamente agli ambienti politici ed economici, mentre l'opinione pubblica italiana è ancora poco conscia delle potenzialità del vicino mediterraneo.

Il testo è completato dall'ottima prefazione di Sergio Romano e dalla Postfazione di Federico Ghizzoni, a.d. di Unicredit, a cui si aggiunge una bibliografia di massima, funzionale all'approfondimento di alcuni dei temi trattati. Unica pecca, ma veniale se si 
considera l'approccio alla materia e i destinatario dell'opera - il grande pubblico -, sono le imprecisioni relative alla storia ottomana ottocentesca, troppo spesso proposta attraverso una visone orientalista ed eurocentrica. Questo, nondimeno, non toglie all'ambasciatore Marsili il grande merito di essere riuscito a descrivere, finalmente senza pregiudizi, la realtà di un Paese, complicato e dinamico, come la Turchia.

Questo saggio si contraddistingue per la sua lucidità analitica, per la sua concretezza rappresentativa e per la sua attualità. Il testo di Marsili non è teorico, ma grazie alla narrazione di ricordi personali e alla costante descrizione di soggetti socio-politici reali e viventi, dona maggior spessore e profondità al testo e ai temi in esso trattati.

Tuttavia, il saggio di Marsili non è una mera descrizione della realtà turca, ma al contrario è l'esposizione dell'essenza di un popolo complesso e ancora diviso nel suo animo tra Oriente e Occidente. Il libro qui presentato è, in altri termini, una fotografia viva e in movimento di una società dinamica e in costante espansione, la quale, sebbene continui ad ammirare l'Europa e l'Italia in modo particolare, si sta rendendo conto di avere le forze necessarie per fare da sé. Questa consapevolezza, evidente nelle parole di Marsili, è anche alla base di molte scelte attuali dei governi turchi, troppo spesso poco comprese dalle diplomazie europee.

I tentennamenti e i dubbi europei, infatti, stanno costringendo Ankara a scegliere e sperimentare vie nuove in politica estera e non solo. Il nuovo ministro degli esteri Davutoglu ne è ben consapevole, soprattutto quando afferma che "“il successo dell'Unione Europea sta nella sua capacità di aprirsi. Se l'Unione Europea si trincererà dietro muri di Berlino culturali, si condannerà da sola al declino"» ${ }^{3}$.

Sebbene il tema dell'adesione all'Europa, come più volte sottolineato, sia ripreso più volte, non è il tema centrale del saggio, che resta senza alcun dubbio la Turchia e la sua società civile e politica. Marsili fornisce un quadro completo del popolo turco, chiaro e comprensibile, ottimo per migliorare nell'opinione pubblica italiana, ancora troppo digiuna di questi argomenti, l'immagine dei Turchi.

L'ambasciatore Marsili, alla domanda perché essere filo-turchi, è solito rispondere in questo modo:

«Perché, se a qualcuno capitasse di trovarsi in una località anche sperduta della Turchia e gli si presentasse un problema grave di qualsiasi genere, avrebbe la 
matematica certezza che chiunque si trovasse per caso nei paraggi gli presterebbe aiuto. $\mathrm{E}$ a nessuno salterebbe in mente di chiedere una ricompensa» ${ }^{4}$.

4 Ibidem, p. 21. 


\section{* L'autore}

Luca Zuccolo, dottorando (PhD Student) in Storia Contemporanea del SUM di Napoli sta sviluppando una ricerca sulla stampa francofona ottomana e la sua rappresentazione dell'Impero d'Oriente. Già dottore magistrale in Storia d'Europa (Bologna 2008), si è occupato della modernizzazione dell'Impero Ottomano, del confronto/scontro tra modernità e tradizione in questo contesto cosmopolita e dello sviluppo dei movimenti sociali che hanno preparato l'avvento della società turca contemporanea.

URL: < http://www.studistorici.com/2010/12/o1/luca-zuccolo >

\section{Per citare questo articolo:}

ZUCCOLO, Luca, «RECENSIONE: Carlo MARSILI, La Turchia bussa alla porta. Viaggio nel paese sospeso tra Europa e Asia, Milano, Bruno Mondadori, 2011, 292 pp.», Diacronie. Studi di Storia Contemporanea: Le sembianze di Clio: approcci alla storia, 29/10/2011,

URL: < http://www.studistorici.com/2011/10/29/zuccolo2_numero_8 >

\section{Diacronie Studi di Storia Contemporanea $\beta$ www.diacronie.it}

Risorsa digitale indipendente a carattere storiografico. Uscita trimestrale. redazione.diacronie@hotmail.it

Comitato di redazione: Marco Abram - Giampaolo Amodei - Jacopo Bassi - Luca Bufarale - Alessandro Cattunar - Alice De Rensis Barbara Galimberti - Deborah Paci - Fausto Pietrancosta - Martina Sanna - Matteo Tomasoni - Luca Zuccolo

Diritti: gli articoli di Diacronie. Studi di Storia Contemporanea sono pubblicati sotto licenza Creative Commons 2.5 Possono essere riprodotti a patto di non modificarne i contenuti e di non usarli per fini commerciali. La citazione di estratti è comunque sempre autorizzata, nei limiti previsti dalla legge. 